\title{
Lithotripsy of pancreatic stones in a patient with cystic fibrosis: Successful treatment of abdominal pain
}

\author{
AA WEISS, MD, FRCPC, JM GREIG, MD, FRCPC, S FACHE, MD, FRCPC
}

AA WEISS, JM GREIG, S FACHE. Lithotripsy of pancreatic stones in a patient with cystic fibrosis: Successful treatment of abdominal pain. Can J Gastroenterol 1992;6(1):25-28. Endoscopic pancreatic sphincterotomy and removal of pancreatic stones has been helpful in selected cases of patients with chronic pancreatitis. This article reports the case of an 18-year-old native Indian woman with cystic fibrosis who was experiencing pain related to pancreatitis, complicated by pancreatic duct stricture and lithiasis. Subsequent dilation of the pancreatic stricture and lithotripsy of the pancreatic ductal stones successfully eliminated the abdominal pain.

Key Words: Abdominal pain, Cystic fibrosis, Lithotripsy, Pancreatic stones

\section{Lithotritie effectuée chez une patiente atteinte de mucovis- cidose: Traitement réussi des douleurs abdominales}

RESUME: Une sphinctérotomie endoscopique et l'élimination des calculs pancréatiques s'avèrent utiles dans certains cas de pancréatite chronique. Le présent article décrit le cas d'une autochtone de 18 ans atteinte de mucoviscidose et qui souffrait de douleurs suite à une pancréatite compliquée par une sténose du canal pancréatique de Wirsung et une lithiase. La dilatation de la sténose et une lithotritie ont permis d'éliminer les douleurs abdominales.

University Hospital-Shaughnessy Site, Vancouver General Hospital, Vancouver, British Columbia

Correspondence and reprints: Dr Alan A Weiss, Department of Medicine, G-538 Jean Matheson Pavilion, University Hospital-Shaughnessy Site, 4500 Oak Street, Vancouver. British Columbia V6H $3 \mathrm{NI}$

Received for publication September 17, 1991. Accepted December 20, 1991
E NDOSCOPIC PANCREATIC SPHINCterotomy and removal of pancreatic stones has been helpful in selected cases of patients with chronic pancreatitis. The case of a patient with cystic fibrosis who had successful relief of abdominal pain related to chronic pancreatitis by means of dilation of the pancreatic stricture and lithotripsy of the pancreatic ductal stones is reported. To the authors' knowledge, this is the first report of successful pancreatic lithotripsy in a patient with cystic fibrosis.

\section{CASE PRESENTATION}

An 18-year-old native Indian woman with cystic fibrosis was admitted to hospital in May 1989 for treatment of pulmonary infection. While hospitalized, epigastric pain developed and persisted for two weeks but subsequently resolved. When the patient was readmitted in August 1989 because of recurrent upper respiratory infection, she also complained of intense and persistent epigastric pain which was worse after meals. The patient also reported a 


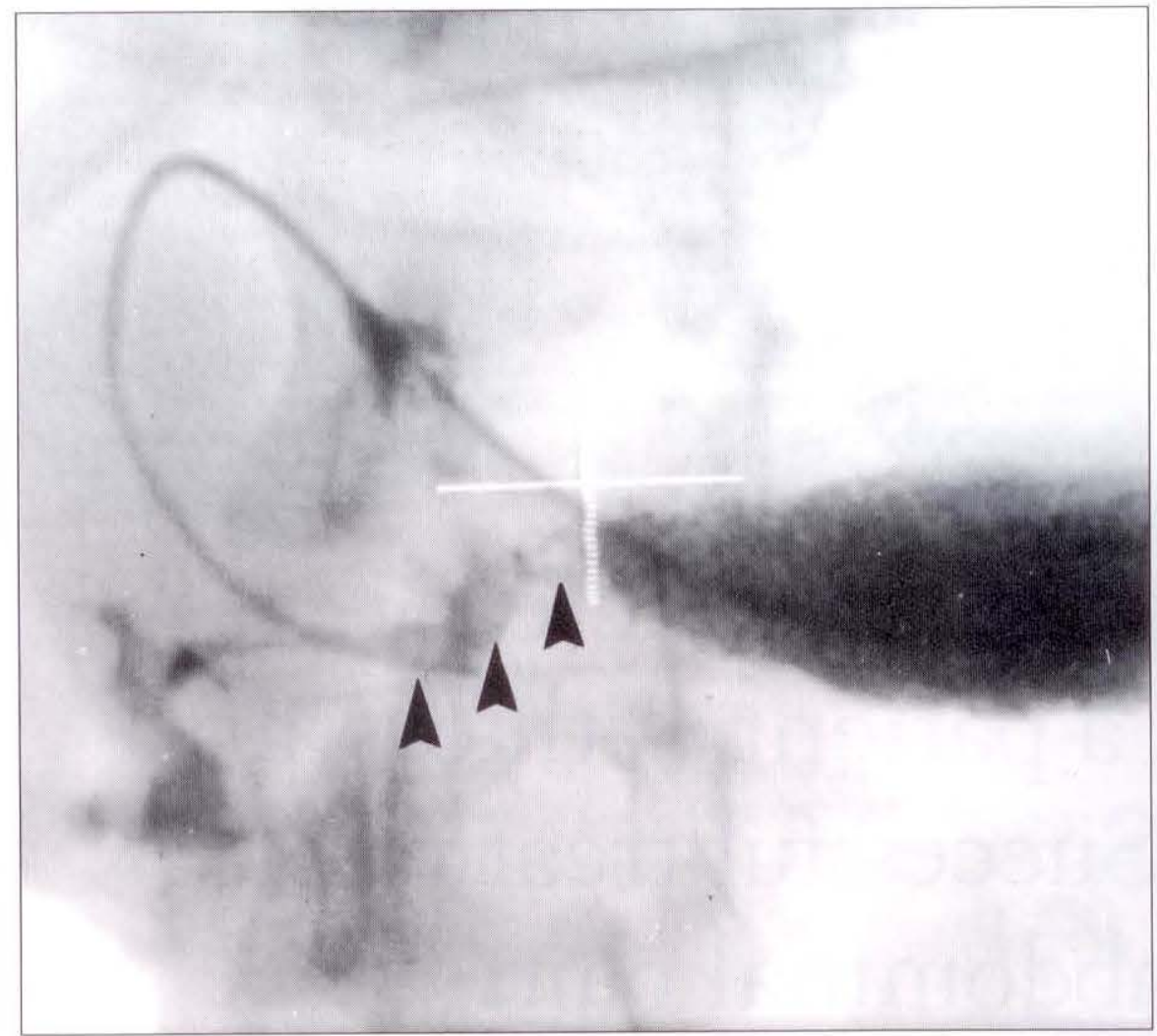

Figure 1) Pancreatic duct stones (arrows) after opacification via nasopancreatic drain

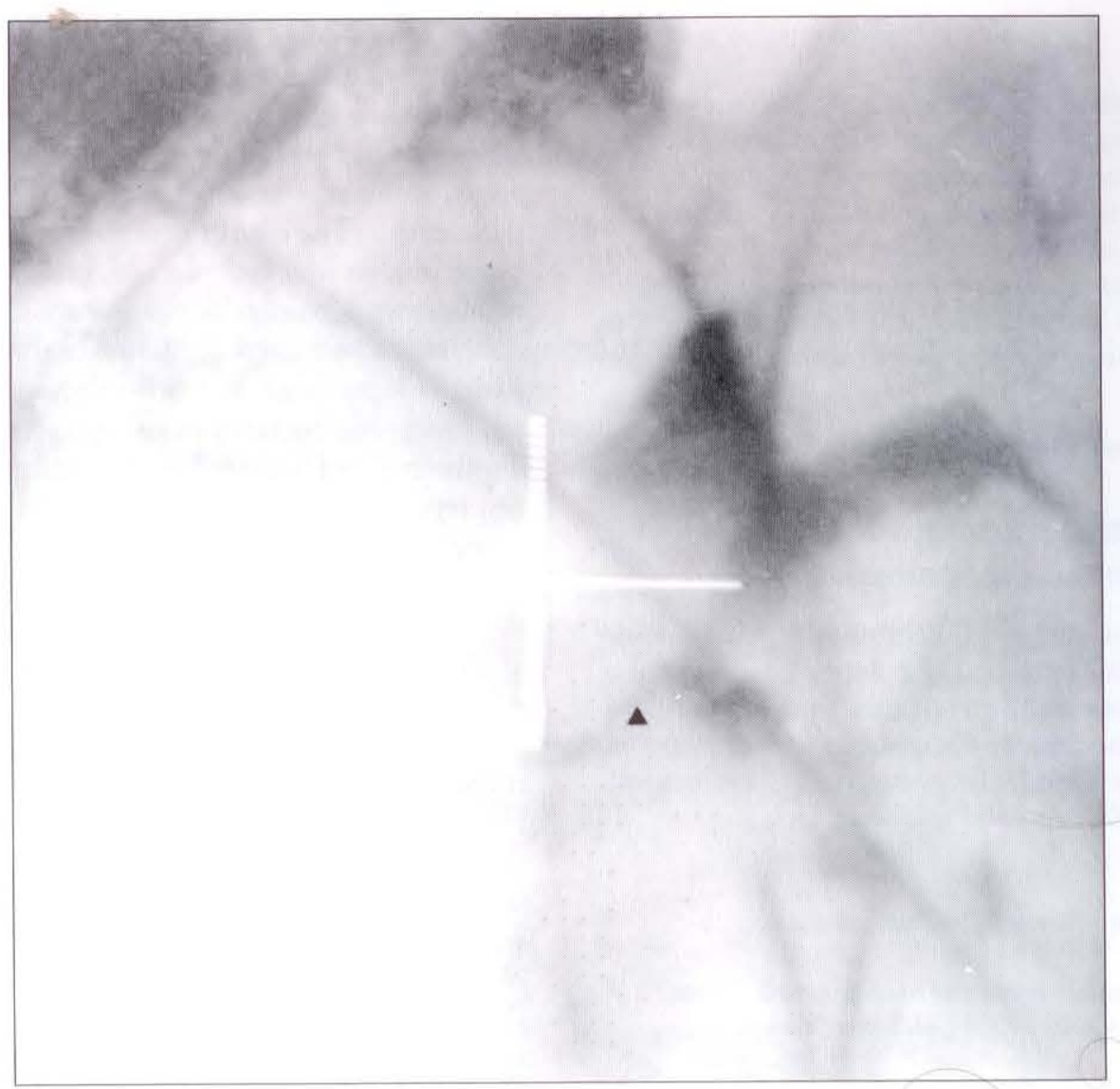

Figure 2) Appearance of pancreatic duct (arrow) after clearance of stone by lithotripsy decreased appetite with a gradual weight loss of approximately $4.5 \mathrm{~kg}$. There was a history of intermittent alcohol abuse.

Upon admission, physical examination revealed moderate respiratory distress and abdominal tenderness in the epigastrium but no evidence of masses or organomegaly. Hematological investigations revealed a mildly elevated white blood cell count of $12,100 / \mathrm{mm}^{3}$ with 9400 granulocytes, an elevated platelet count of $600,000 / \mathrm{mm} 3$ and a hemoglobin of $115 \mathrm{~g} / \mathrm{L}$. Calcium was $2.19 \mathrm{mmol} / \mathrm{L}$ (normal 2.12 to 2.62 ) and amylase less than $30 \mathrm{IU} / \mathrm{L}$. Liver enzyme studies indicated normal aspartate aminotransferase, slightly elevated gamma glutamyltransferase of 45 IU (normal 10 to 40), and elevated alkaline phosphatase of 179 IU (normal 29 to 133). Sputum culture grew Psendomonas aeruginosa, and antibiotic treatment with tobramycin and ceftazidime was begun.

During hospitalization, abdominal pain progressively worsened, radiated to the back and became exacerbated after meals. Initial investigations of an upper gastrointestinal series and an upper gastrointestinal endoscopy revealed no abnormality. Plain film of the abdomen was normal. Abdominal ultrasound disclosed slight dilation of the proximal pancreatic duct and a subsequent endoscopic retrograde cholangeopancreatography (ERCP) showed a normal biliary tree but an abnormal pancreatic duct with proximal stricture and dilation behind this short initial narrowing. The dilated segment was about $2 \mathrm{~cm}$ in length and contained five small stones.

The patient was treated conservatively with lipancreatin (Cotazyme; Organon Pharmaceuticals) and pain controlled with meperidine administered several times daily. These medications were continued following discharge in September 1989.

Persistence of abdominal pain as well as recurrent pulmonary infection necessitated a third admission in January 1990. While hospitalized, the patient underwent ERCP using a Pentax duodenoscope FD $34 \mathrm{H}$ during which a sphincterotomy of the pancreatic duct was performed using an Olympus standard full type papillotome 
and the pancreatic duct stricture dilated by passage of a standard steeltipped Cook cannula. A good flow of dye was achieved in and out of the pancreatic duct. Attempts to remove the pancreatic stones with basket and balloon catheters were unsuccessful. Due to retching and respiratory distress, it was not possible to insert a nasopancreatic drain so the procedure was concluded.

The abdominal pain worsened and continued without relief for several days following the procedure. As a result of postprandial nausea and vomiting and further decrease in weight, total parenteral nutrition was commenced but was complicated by candida sepsis. The total parenteral nutrition line was removed and the catheter tip was cultured and grew Candida albicans. Therapy was initiated with amphotericin B but progress was further complicated by another septic episode in which Staphylococcus coagulasepositive species and Streptococcus viridans were isolated from blood. These organisms were also cultured from a small abscess on the dorsal aspect of the patient's right hand - site of a previous intravenous line. The patient was treated with vancomycin and ceftazidime and following recovery was discharged home in April 1990.

The patient continued to be seen on an outpatient basis for about one month. During this time, she experienced recurrent abdominal pain that was later complicated by the development of high fever, worsening cough and sputum production necessitating re-admission to hospital one month later. At that time, she was in respiratory distress and chest examination revealed course crepitations in both lungs, frequent cough and mild dyspnea. The patient was febrile with a regular pulse rate of 140 beats $/ \mathrm{min}$. Blood cultures were positive for multiple organisms including Ps aeruginosa, staphylococcus coagulase-negative species, and Strep viridans. Treatment with tobramycin and vancomycin produced a satisfactory response.

While in hospital the patient continued to experience abdominal pain, nausea and vomiting; therefore, when she was afebrile with no evidence of sepsis, ERCP was repeated during which a nasopancreatic drain was inserted into the pancreatic duct. Visualization of the pancreatic duct through the nasopancreatic drain was difficult because of leakage of dye through the multiple holes within it preventing dye from entering the pancreatic duct itself. This problem was solved by passing an open ended interventional angiographic guidewire through the nasopancreatic catheter and injecting dye through it, thus enabling visualization of the pancreatic duct. Five small radiolucent stones were noted in the proximal part of the dilated pancreatic duct (Figure 1).

The stones were targeted with the help of fluoroscopy and 4000 shocks administered reaching up to $18.1 \mathrm{kV}$ energy level. Following the lithotripsy, a pancreatogram showed clearance of the stones from the duct (Figure 2). The nasopancreatic tube was removed the following day. The pain was completely resolved, meperidine was discontinued over a period of several days. The patient was discharged on a low fat diet and lipancreatin (three tablets with meals). The patient was advised to abstain from alcohol but she continued to drink intermittently. There was no significant pain for approximately six months; however, subsequently there was occasional recurrence of abdominal pain. These episodes were milder compared with the abdominal pain experienced prior to lithotripsy of the pancreatic stones. It was felt that these episodes of pain may have been induced by alcohol intake.

The patient had four further admissions over the next 12 months for treatment of pulmonary infections and on the last admission she suffered cardiorespiratory arrest and died. At autopsy, the pancreas was found to be small, there was extensive fibrosis and fat was present. The acini were greatly reduced in number. The main pancreatic duct was dilated and mild periductal inflammation was present, no pancreatic stones were found.

\section{DISCUSSION}

Abdominal pain is a frequent complaint in patients with cystic fibrosis, but the severity and location of the pain vary depending on the underlying cause (1). Abdominal pain due to pancreatitis is a known complication of cystic fibrosis.

Pancreatitis may be an initial presentation before other manifestations of cystic fibrosis or it may occur during the course of an established illness (2). Damage to the pancreas in patients with cystic fibrosis is characterized by fibrosis, fatty replacement and cysts. The pancreatic lesions are caused mostly by obstruction of small ducts by secretions and debris. Mild inflammation around the obstructed acini may be present and stenosis of the large pancreatic ducts may occur. Formation of ductal stones may be secondary to pancreatitis and stasis of pancreatic secretions; their presence may further aggravate the ductal obstruction and stasis.

Effective treatment of pain due to chronic pancreatitis is very difficult with patients frequently requiring symptomatic pain relief with narcotic analgesics. Pancreatic enzymes may be used in the treatment of abdominal pain in patients with chronic pancreatitis. The mechanism of action is thought to be decreased stimulation of the pancreatic secretions (3). This therapy did not provide any relief from pain in the present patient.

Endoscopic sphincterotomy with removal of pancreatic stones has been used successfully in treating abdominal pain in selected patients with chronic pancreatitis $(4,5)$. However, in the present patient, pancreatic sphincterotomy and dilation of the stricture of the pancreatic duct produced no significant relief of pain. The subsequent insertion of a nasopancreatic drain through the sphincterotomy allowed the pancreatic duct to be opacified and successful lithotripsy (6) of the pancreatic stones to be performed. This measure proved to be therapeutic for the patient with successful relief of abdominal pain.

The possibility of pancreatitis and pancreatic lithiasis should be considered in a patient with cystic fibrosis who presents with abdominal pain characteristic of pancreatitis. Treatment of pancreatic lithiasis, as demonstrated in the present patient, may lead to significant relief of abdominal pain. 
ACKNOWLEDGEMENTS: The authors thank Dr EM Nakielna and Dr D Burdge for referral of the patient.

\section{REFERENCES}

1. Barbero GJ. Cystic fihrosis. In: Bockus HL, ed. Gastroenterology, vol 3.

Philadelphia: Saunders, 1976:1170-9.

2. Masaryk T], Achkar E. Pancreatitis as initial presentation of cystic fibrosis in young adults: A report of 2 cases. Dig Dis Sci 1983;28:874-8.

3. Vyas H, Matthew DJ, Millar PJ. A comparison of enteric coated microspheres with enteric coated tablet pancreatic enzyme preparation in cystic fibrosis: A controlled study. Eur J Pediatr 1990;149:241-3.

4. Huibregtse K, Schneider B, Vrig AA, Tytgat GN. Endoscopic pancreatic drainage in chronic pancreatitis.
Gastrointest Endosc 1988:34:9-15.

5. Ponsky JL, Doppler DW. Endoscopic sphincterotomy and removal of pancreatic duct stones. Am Surg 1987;53:613-6.

6. Sauerbruch T, Holl J, Sackmann M, Werner R, Wotzka R, Paumgartner G. Disintegration of pancreatic duct stone with extracorporeal shock waves in a patient with chronic pancreatitis. Endoscopy 1987;19:207-8. 


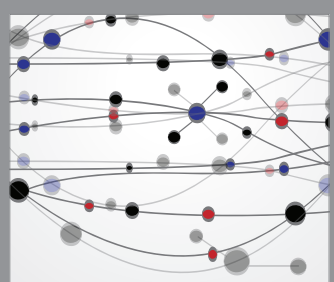

The Scientific World Journal
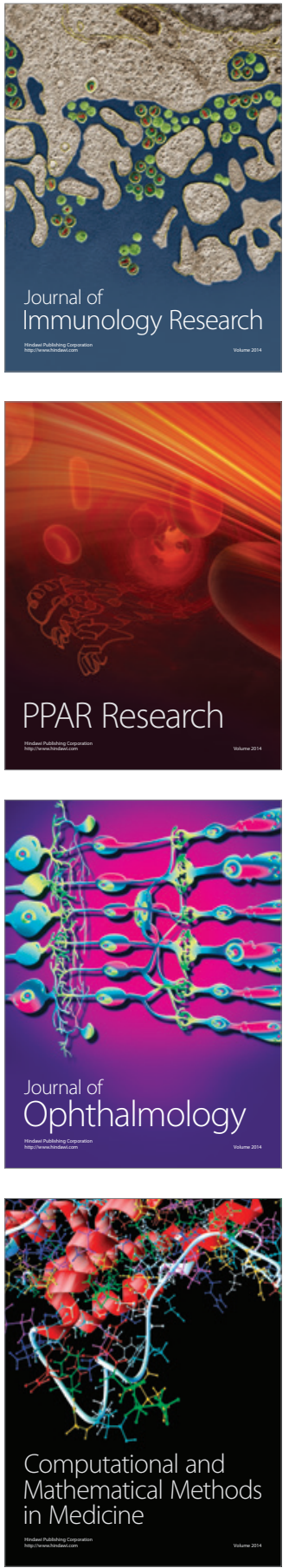

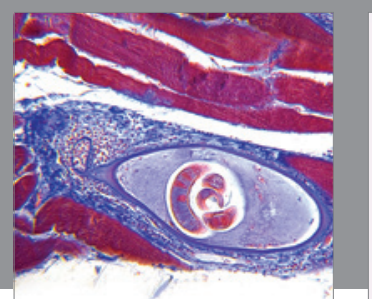

Gastroenterology Research and Practice

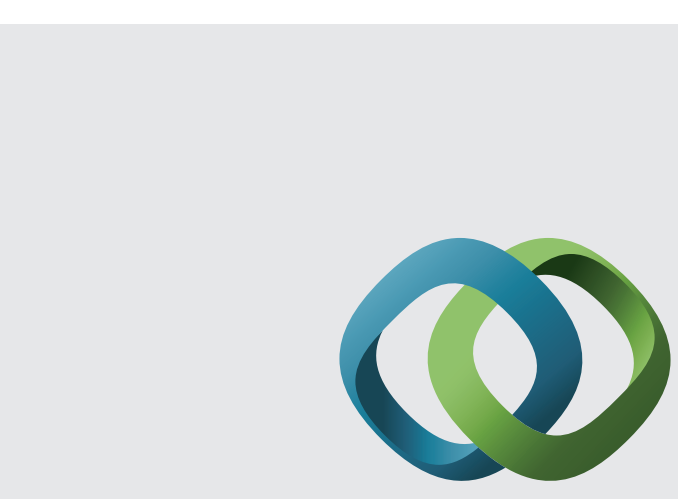

\section{Hindawi}

Submit your manuscripts at

http://www.hindawi.com
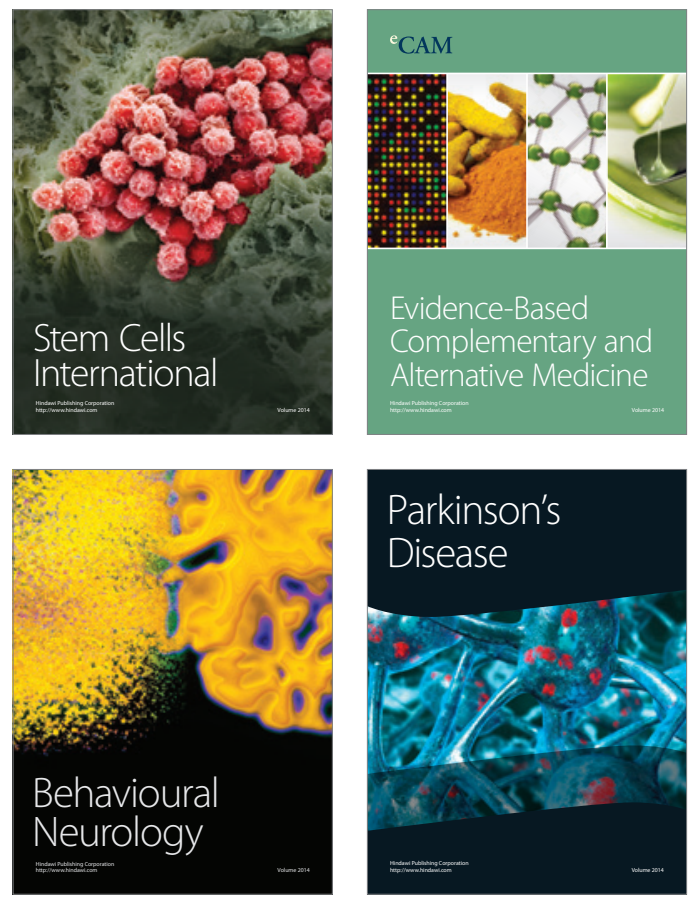
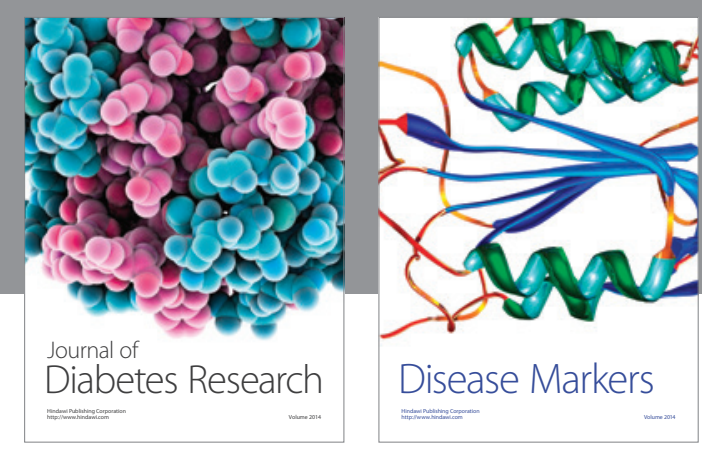

Disease Markers
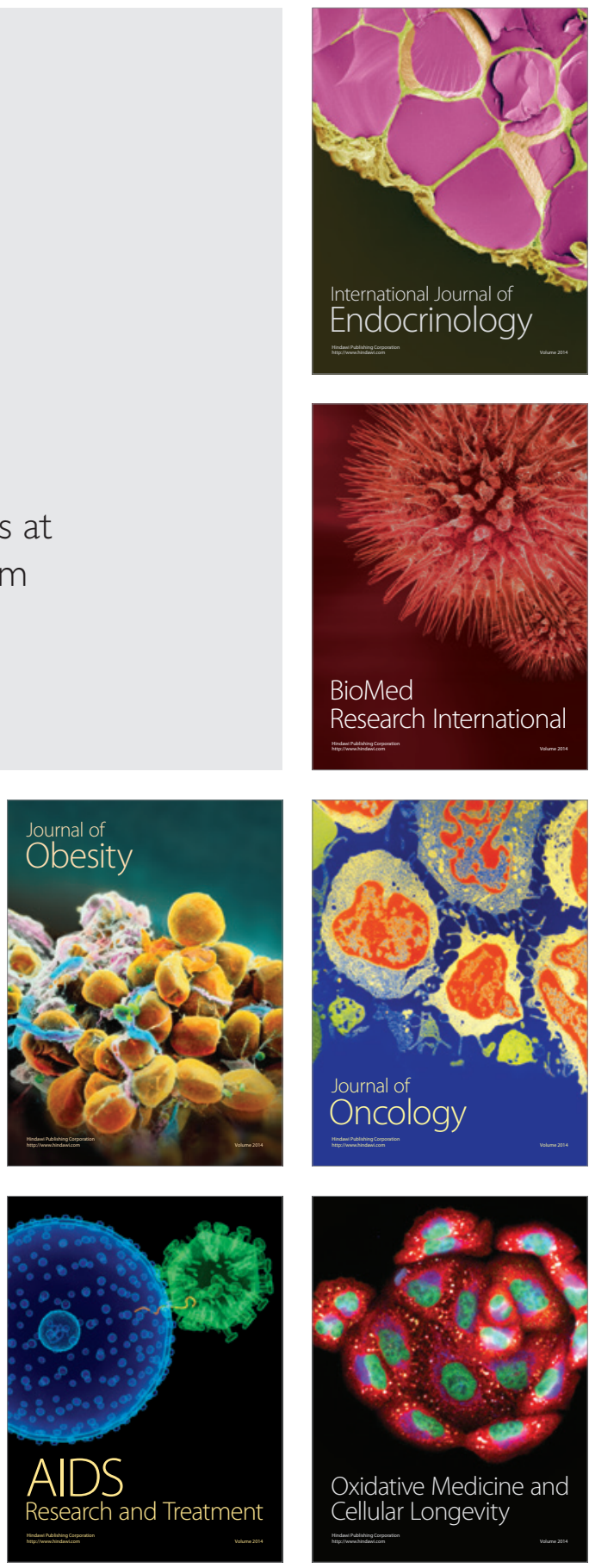